\title{
Dynamic metabolic profiling in vegetable soybean seed development
}

\author{
Runfang Hu\#, Yumei Zhang\#, Yuhua Chen, Guoqiang Lin* \\ Institute of Crop Sciences, Fujian Academy of Agricultural Sciences, Fuzhou 350013, P.R. China \\ \#These authors contributed equally in this work
}

\section{A B S T R A C T}

\begin{abstract}
The edible quality of vegetable soybean is significantly associated with the number and type of metabolites. In the present study, metabolites in new vegetable soybean variety Mindou 6 at 49 to 67 days after flowering was investigated using gas chromatography coupled with mass spectrometry. The results showed that a total of 102 metabolites were identified from the vegetable soybean seeds, including 36 organic acids (35.3\%), 21 amino acids, 11 polyols, 9 sugars, 7 phosphoric acids, 5 amines, 4 fatty acids, 4 nucleotides, and 5 other components. The principal component analysis indicated that the metabolites of amino acids, organic acids and sugars were greatly related to the quality of the vegetable soybean at the filling stages. A total of 40 differential metabolites were identified, including 14 amino acids, 13 organic acids and 2 sugars, and were found to be mainly involved in the metabolism of glycolysis, amino acids and phenylpropanoid. We systematically analyzed the change in the contents of metabolites in the developmental seeds of Mindou 6 and its effects on seed nutrition and eating quality. The suitable picking time was determined by combining the fresh pod yield and appearance quality. Taken together, these results can provide a theoretical reference for vegetable soybean breeding for high quality and efficient production.
\end{abstract}

Keywords: Gas chromatography coupled with mass spectrometry (GC-MS); Metabolites; Vegetable soybean

\section{INTRODUCTION}

The vegetable soybean, called "Edamame" in Japan or "Mao Dou" in China, is harvested at the reproductive stage ranging between $\mathrm{R} 6$ and $\mathrm{R} 7$ when seeds are immature and pods are not transitioning to a yellow color (Mimura et al., 2007; Czaikoski et al., 2013). This soybean is an excellent source of proteins, free amino acids, carbohydrates, dietary fibers, vitamins, minerals and phytoestrogens (Song et al., 2003), and has developed into a popular market as a large vegetable (Duppong and Hatterman-Valenti, 2013; Zhang et al., 2013). The most obvious features in appearance quality are large pods and seeds, and a green color. As eating quality is an important factor on influencing market share, the standard of eating quality for vegetable soybeans demands a taste that is fragrant, sweet, soft and waxy. Moreover, the most important eating quality for vegetable soybean is that it is sweet and possesses a umami experience in taste. The content of soluble sugar, starch, fat and free amino acid is higher in the vegetable soybean, as evident by the sweeter taste, waxy and soft texture, and a stronger umami (Zhang et al., 2006). The selection of the suitable germplasm of vegetable soybean could provide a good source of nutrition for diet. The chemical variability linked with the eating quality of vegetable soybean, which mainly includes high content of soluble sugar, free amino acids and organic acids. This study focused on the effects of sugar, starch, protein, amino acids on eating quality of vegetable soybean (Zhang et al., 2006; Wang and Wang, 2002), systematic studies on the type and number of metabolites affecting eating quality have rarely been reported.

The metabolite is the final product of gene expression, and the plant phenotype is significantly associated with the number and type of metabolites (Fiehn, 2002). Using metabolism, it is helpful for high throughput to explore the metabolites closely related to plant growth and development. With the emergence of gene expression, metabolites were used to further explain the molecular mechanism of plant growth and development. In recent years, with the development of mass spectrometry and analytical techniques, metabolomics is widely used and has been accepted as an innovative tool (Shepherd et al., 2011; Tohge et al., 2011). A diverse set of antioxidant metabolites,

\footnotetext{
*Corresponding author:

Guoqiang Lin, Institute of Crop Sciences, Fujian Academy of Agricultural Sciences, Fuzhou 350013, P.R. China. Fax: +86 59187573144. Tel: +86 591 87589907. E-mail: Runfang Hu: 279071648@qq.com, Yumei Zhang: zym1122@126.com, Yuhua Chen: 550189102@qq.com, Guoqiang Lin: lgq308@163.com.
} 
including tocopherols, flavonoids, phenylpropanoids, and ascorbate precursors, which are likely responsible, at least in part, for the greater tolerance of vegetable soybeans to high temperatures during seed development, were found to be enriched in seeds of the heat tolerant genotype (Chebrolu et al., 2016). Using metabolomics to study Brassica napus and Brassica campestris, the results showed that a higher content of polyunsaturated fatty acids and sucrose were observed in turnip rape, while the overall oil content and sinapine levels were higher in the oilseed rape (Kortesniemi et al., 2015).

To date, there are many vegetable soybean varieties bred and cultivated in China. Until the present study was published, there were no comprehensive studies available that reported the dynamic metabolic profiling in vegetable soybean filling seeds. In this study, the metabolites of vegetable soybean seeds with high yield and good quality were analyzed at various time points to assess the metabolic levels in order to improve the quality of vegetable soybeans and provide theoretical guidance.

\section{MATERIALS AND METHODS}

\section{Plant material}

Vegetable soybean (Glycine max) 'Mindou 6' was newly bred by the Institute of Crop Sciences, Fujian Academy of Agriculture Science, and approved by crop variety approval committee of Fujian province in 2013. In autumn 2014, soybean Mindou 6 plants were grown in the experimental field. To investigate the dynamic metabolites profiles of the seeds at the filling stage, the flowering days of 100 plants were tagged. Only the pods of similar size from the same part of the tagged plants were harvested, and a total of 20 pods from 20 different plants were mixed as a sample. Pods including seeds were sampled at 6-day intervals from initiation of seed formation to suitable to eat as vegetable soybean. Pods appeared light yellow at 67 days after flowering (DAF). At the whole seed-filling period (DAF49 67), the color of seeds had no significantly difference. At 49, 55, 61 and $67 \mathrm{DAF}$, developing pods including seeds were harvested and stored in liquid nitrogen until used. Meanwhile, 100 similarly sized pods with the same sampling day were used to attain the 100-fresh-pod weight.

\section{Metabolite extraction}

Seed sample extraction was performed as previously described with slight modification (Lisec et al., 2006). Samples were extracted with methanol, followed by the addition of Ribitol $\left(0.2 \mathrm{mg} \cdot \mathrm{mL}^{-1}\right.$ stock in $\left.\mathrm{ddH}_{2} \mathrm{O}\right)$ as an internal quantitative standard. Then chloroform were added, followed by centrifugation at 2,200 $\times g$ for $10 \mathrm{~min}$ to obtain a clear solution. The supernatant was transferred and dried under moderate nitrogen. The dried samples were dissolved in methoxyamine pyridine $\left(15 \mathrm{mg} \cdot \mathrm{mL}^{-1}\right.$ solution). Lastly, $60 \mu \mathrm{L}$ of an MSFTA reagent (containing 1\% TMCS) was added and incubated for $60 \mathrm{~min}$ at $25{ }^{\circ} \mathrm{C}$. The tube was then centrifuged for $10 \mathrm{~min}$ at $12,000 \times g$. Retention indices were calibrated with the addition of a C8-C20, C21-C40 n-alkane mixture to each sample (Sanchez et al., 2012). To avoid the systematic error, all of the samples were randomly sampled and processed at once. All experiments were processes in batches and repeated six times.

\section{GC-MS analysis}

The extracted samples were analyzed using an Agilent 7890A GC system coupled to an Agilent 5975C intert XL EI/CI mass spectrometric detector (MSD) system (Agilent Technologies, Santa Clara, CA, USA). Gas chromatography was performed on an HP-5MS capillary column ( $5 \%$ phenyl methyl silox: $30 \mathrm{~m} \times 250 \mu \mathrm{m}$ i.d., $0.25 \mu \mathrm{m}$ film thickness, Agilent J \& W Scientific, Folsom, CA, USA) to separate the derivatives. One microliter of derivatized sample was injected with a 20:1 split injection ratio. The injection temperature was $280{ }^{\circ} \mathrm{C}$, the interface was set to $150{ }^{\circ} \mathrm{C}$ and the ion source was adjusted to $250^{\circ} \mathrm{C}$. The temperature gradient program was as follows: initial temperature set at $80{ }^{\circ} \mathrm{C}$ for $5 \mathrm{~min}$, followed by a $20^{\circ} \mathrm{C} \cdot \mathrm{min}^{-1}$ rate increase up to $300^{\circ} \mathrm{C}$ and then maintaining the temperature at 300 ${ }^{\circ} \mathrm{C}$ for $6 \mathrm{~min}$. The carrier gas (helium) achieved a constant linear velocity at $1 \mathrm{~mL} \cdot \mathrm{min}^{-1}$. Mass spectrometry was determined by the full-scan method ranging from 35 to 780 $(\mathrm{m} / \mathrm{z})$ at the scan speed of $1,000 \mathrm{u} / \mathrm{s}$. The ionization mode displayed an electron impact at $70 \mathrm{eV}$ and the detector voltage was $0.9 \mathrm{KV}$.

\section{Metabolites identification and statistical analyses}

Peaks with the signal to noise ratio $>6$ were picked-up by Agilent ChemStation. Raw GC/MS data were converted into CDF format (NetCDF) using Agilent GC/MS 5975 data analysis software and were subsequently processed by the XCMS (www.bioconductor.org) using XdCMS default settings with the following changes (Vanholme et al., 2012): $x \mathrm{cmsSet}$ (fwhm $=3$, snthresh $=3, \max =300$, mzdiff $=0.5$, step $=0.1$, steps $=2)$, rector $($ method $=" l i n e a r "$, family="gaussian", plottype="mdevden") and bandwidth (bw) of 5. The data were normalized on the basis of the abundance of the internal standard and transformed with unit variance scaling. Identification of metabolites in samples was performed by searching in two databases, namely the National Institute of Standards and Technology (NIST) database and the Golm Metabolome Database (GMD) (Kopka et al., 2005).

Multivariate statistical analyses such as principal component analysis (PCA) and partial least squares-discriminant analysis (PLS-DA) were carried out using Soft Independent Modeling of Class Analogy (SIMCA)-P (version 11.0, 
Umetrics AB, Umea, Sweden). Any notable differences between the metabolites were screened by the loading plot in PLS-DA. The variables with VIP (Variable Importance in the Projection) values greater than 0.8, which played significant roles in the classification, were selected for further analysis. Subsequently, independent $t$-test was used for excluding the variables that were not significantly different $(P>0.05)$.

\section{RESULTS}

\section{Phenotype of pod and seed in different periods}

After entering the reproductive growth stage, the pod length and width of the vegetable soybean develop first, and then the size and fresh weigh of seeds begins to develop until its pod length and width arrive to its inherent size. The fresh 100-pod weight of Mindou 6 was 121.30, 181.24, 221.86 and $230.12 \mathrm{~g}$ at four different stages, respectively, which showed that the fresh pod weight increased gradually with a tendency of fast first and then slow. Compared to DAF49, fresh pods weighed at at DAF55, DAF61 and DAF67 were $49.41 \%, 82.90 \%$ and $89.71 \%$ higher, respectively. Pods appeared light yellow at DAF67. At the whole seed-filling period (DAF49-DAF67), the color of the seeds was not significantly different. Taken together, the suitable period to pick Mindou 6 was found to be between DAF61 and DAF67, and the harvest must be completed before DAF67.

\section{Metabolites changes during seed development}

Three hundred and fifteen peaks were detected, 102 of which could be specifically identified by the NIST and GMD library and used for further statistical analysis. These metabolites could be nine categories of chemicals, including organic acid, amino acid, polyol, sugar, phosphoric acid, amine, fatty acid, nucleotide and other. Thirty-six species in organic acids accounted for $35.3 \%$, followed by 21 kinds of amino acids accounting for $20.6 \%, 11$ kinds of polyols accounted for $10.8 \%, 9$ sugars for $8.8 \%, 7$ phosphoric acids for $6.9 \%, 5$ amines for $4.9 \%, 4$ kinds of fatty acids and nucleotides respectively, each accounted for $3.9 \%$, and 5 other substances accounted for $4.9 \%$.

Principal component analysis (PCA) was used to visualize the developmental patterns of the seeds and changes in concentrations, with a total of 102 metabolites, of the vegetable Mindou 6 seeds. Four different stages were used for analysis and six repeats, with exception to the samples at DAF49 due to an abnormal repeat deletion, were implemented. The PCA model revealed a statistically significant $(P<0.05)$ separation of the samples into four groups (Fig. 1). The metabolites profiles at DAF49 and DAF55 were more closely related than those at DAF61 and DAF67, suggesting that a large portion of new metabolites were synthesized after DAF61. The score plot of PCA for the first two components $(\mathrm{R} 2 \mathrm{X}=0.810$, Q2 0.485) showed a separate trend for the vegetable soybean seeds at the developmental stages (Fig. 1). The first two principal components (PCs) explained 52.5\% of the total variance, which PC1 and PC2 accounted for $38.1 \%$ and $14.4 \%$, respectively. The metabolites in vegetable soybean seeds which contributed to PC1 was dominated by amino acids (e.g. asparagine, homoserine, threonine, alanine, serine, isoleucine, leucine, valine, proline and lysine) and organic acid (e.g. tetronic acid, suberyl glycine, 2,3-dihydroxybutanedioic acid, succinic acid, malic acid and fumaric acid), while glucose, sorbitol, fructose, melibiose, isomaltose and sucrose were the main contributors of PC2 (Fig. 2, File S1). The resulting composition analysis suggests that the amino acids,

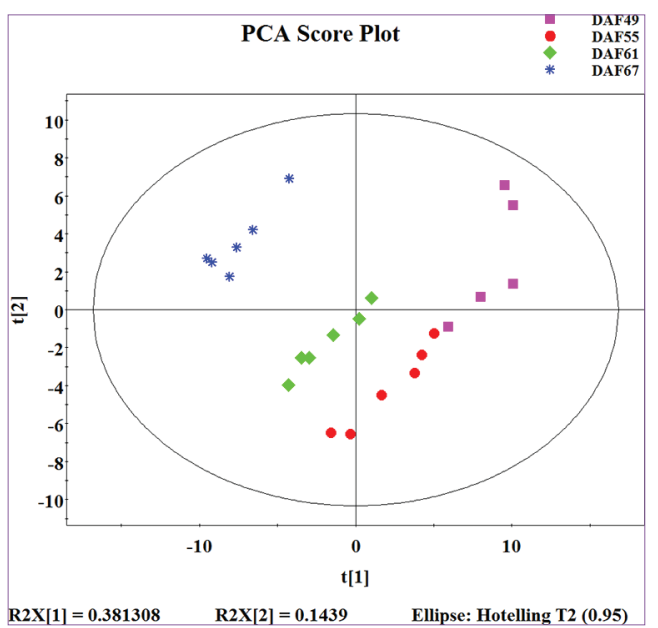

Fig 1. Score of principal component analysis (PCA) of metabolic profiles of vegetable soybean seeds at different stages. Pink squares, red dots, green diamonds and blue asterisks indicate the samples at DAF49, DAF55, DAF61 and DAF67, respectively. The first two principal components (PCs) explained $52.5 \%$ of the total variance, which PC1 and PC2 accounting for $38.1 \%$ and $14.4 \%$, respectively.

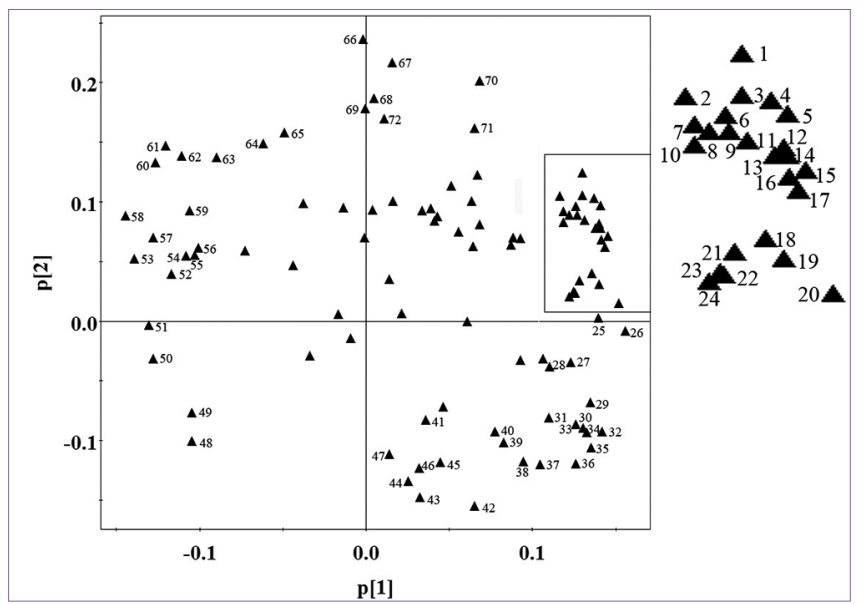

Fig 2. Loading plot of PCA of metabolic profiles of vegetable soybean seeds at different stages. The metabolite numbers are shown in File S1. 
$\mathrm{Hu}$, et al.

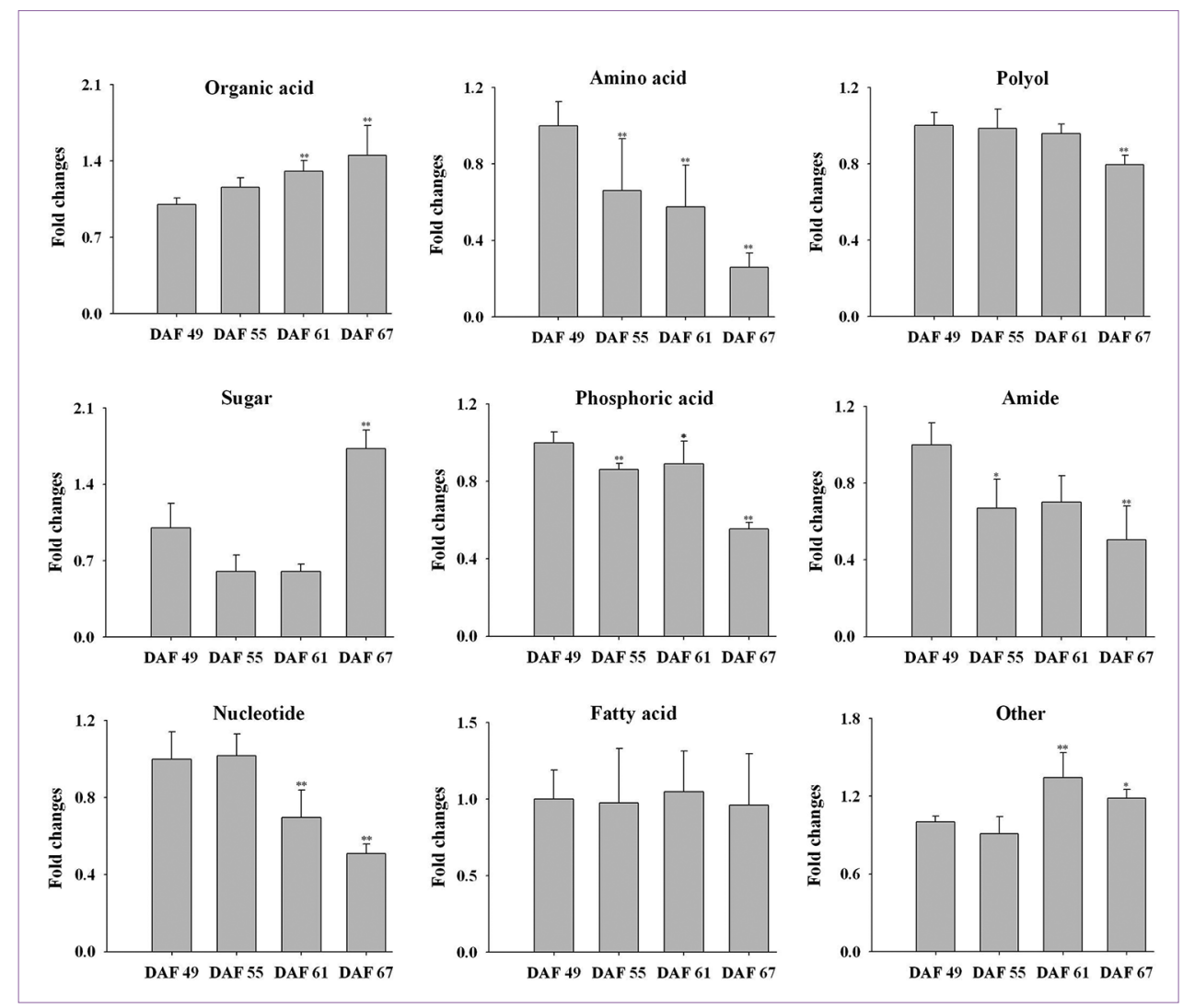

Fig 3. Fold changes in metabolites of vegetable soybean seeds at four developmental stages. Data are presented as mean \pm standard deviation. $\mathrm{DAF}$, days after flowering. Asterisks indicate statistically significant differences compared to DAF $49\left({ }^{*} P<0.05 ;{ }^{*} P<0.01\right)$.

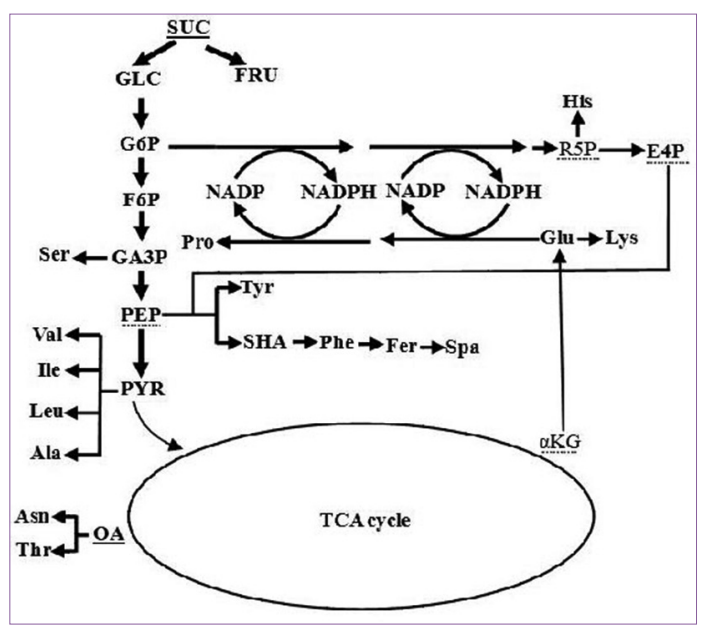

Fig 4. The proposed metabolic pathway of vegetable soybean seeds at different stages. The metabolites with solid lines represent a detectable metabolite; however, the difference is not significant. Dotted lines represent the non-detected metabolites. SUC: sucrose; GLC: glucose; FRU: fructose; G6P: glucose-6-phosphate; F6P: frucose-6-phosphate; GA3P: glyceric acid-3-phosphate; PEP: phosphoenolpyruvate; PYR: pyruvic acid; OA: oxalic acid; $\alpha \mathrm{KG:} \alpha$-ketoglutaric acid; R5P: ribose-5-phosphate; E4P: erythrose-4-phosphate; Ser: serine; Val: valine; lle: isoleucine; Leu: leucine; Ala: alanine; Asn: asparagine; Thr: threonine; His: histidine; Pro: proline; Glu: glutamic acid; Lys: lysine; Tyr: tyrosine; SHA: shikimic acid; Phe: phenylalanine; Fer: ferulic acid; Spa: sinapinic acid. organic acids and carbohydrate metabolites have a great relationship with the quality of vegetable soybean during development.

Further analysis indicated that the content of organic acid, sugar and other metabolites increased gradually with seed development. On the contrary, that of amino acid, phosphoric acid, amine and nucleotide metabolites gradually decreased, and minimum changes was noted in the content of polyol and fatty acids (Fig. 3). Among these nine categories, organic acid at DAF67 exhibited the highest content. However, amino acid content exhibited the highest content at DAF49 and then decreased slowly at DAF55 to DAF61, and decreased rapidly from DAF61 and reached the lowest content at DAF67. In contrast, the levels of sugars were decreased from DAF49 to DAF55 and stayed relatively stable from DAF55 to DAF61. However, the sugar levels dramatically increased again at DAF67, mainly due to a sharp rise in sucrose at DAF67 which the level of sugar shapely increased from 193.01 at DAF61 to 1801.72 at DAF67 (the data was not shown). With the development of seeds, the levels of fructose and glucose first decreased and then increased, and the fructose content was about $6.21-7.68$ times that of glucose (Table 1). 
$\mathrm{Hu}$, et al.

Table 1: Different metabolites isolated from developing seeds

\begin{tabular}{|c|c|c|c|c|c|c|}
\hline \multirow[t]{2}{*}{ Category } & \multirow[t]{2}{*}{ Metabolite } & \multirow[t]{2}{*}{ VIP } & \multicolumn{4}{|c|}{ Content in different stage } \\
\hline & & & DAF 49 & DAF 55 & DAF 61 & DAF 67 \\
\hline \multirow[t]{2}{*}{ Amine } & 2,5-Diaminovalerolactam & 0.864975 & $6.91 \pm 3.20$ & $3.08 \pm 2.38$ & $1.51 \pm 0.88$ & $1.54 \pm 0.87$ \\
\hline & N-Acetyl glucosamine & 0.969397 & $1.16 \pm 0.42$ & $0.52 \pm 0.12$ & $0.36 \pm 0.25$ & $0.38 \pm 0.06$ \\
\hline \multirow[t]{14}{*}{ Amino acid } & Alanine & 0.817621 & $38.04 \pm 8.02$ & $19.28 \pm 11.72$ & $17.05 \pm 14.01$ & $5.58 \pm 3.52$ \\
\hline & Asparagine & 0.917946 & $2554.20 \pm 329.05$ & $1860.33 \pm 651.81$ & $893.07 \pm 351.34$ & $228.96 \pm 127.11$ \\
\hline & Glutamic acid & 1.51271 & $307.21 \pm 153.82$ & $65.65 \pm 25.08$ & $322.78 \pm 144.63$ & $76.19 \pm 26.88$ \\
\hline & Histidine & 0.905464 & $699.10 \pm 358.08$ & $219.20 \pm 253.12$ & $33.45 \pm 40.85$ & $7.78 \pm 11.91$ \\
\hline & Homoserine & 0.969544 & $15.91 \pm 1.16$ & $9.37 \pm 3.47$ & $3.33 \pm 1.69$ & $1.41 \pm 0.58$ \\
\hline & Isoleucine & 0.949397 & $108.46 \pm 28.60$ & $42.30 \pm 24.93$ & $39.53 \pm 24.55$ & $23.02 \pm 11.19$ \\
\hline & Leucine & 0.833701 & $108.38 \pm 36.25$ & $49.90 \pm 34.78$ & $34.51 \pm 25.80$ & $19.69 \pm 11.67$ \\
\hline & Lysine & 0.878405 & $137.13 \pm 62.00$ & $50.80 \pm 40.60$ & $44.61 \pm 36.94$ & $14.51 \pm 11.45$ \\
\hline & Phenylalanine & 1.09651 & $33.40 \pm 4.47$ & $20.02 \pm 5.70$ & $25.80 \pm 7.51$ & $17.71 \pm 4.54$ \\
\hline & Proline & 0.93897 & $165.27 \pm 63.84$ & $52.59 \pm 36.68$ & $38.59 \pm 31.42$ & $16.62 \pm 10.18$ \\
\hline & Serine & 0.801906 & $511.64 \pm 107.70$ & $253.37 \pm 172.53$ & $221.26 \pm 203.09$ & $74.26 \pm 51.73$ \\
\hline & Threonine & 0.839582 & $78.78 \pm 11.15$ & $39.92 \pm 20.94$ & $32.39 \pm 24.93$ & $15.81 \pm 9.45$ \\
\hline & Tyrosine & 1.07158 & $101.86 \pm 19.16$ & $43.52 \pm 15.13$ & $39.48 \pm 15.46$ & $38.93 \pm 9.65$ \\
\hline & Valine & 0.981429 & $239.54 \pm 76.69$ & $81.62 \pm 46.34$ & $58.72 \pm 31.11$ & $34.04 \pm 15.87$ \\
\hline \multirow[t]{13}{*}{ Organic acid } & 2,3-Dihydroxybutanedioic acid & 1.01876 & $14.73 \pm 1.13$ & $8.97 \pm 0.41$ & $8.31 \pm 1.12$ & $5.30 \pm 0.35$ \\
\hline & 2-Aminoadipic acid & 1.51819 & $24.16 \pm 2.33$ & $34.40 \pm 7.85$ & $44.43 \pm 8.30$ & $15.72 \pm 3.57$ \\
\hline & $\begin{array}{l}\text { 4-Hydroxy-3-methoxybenzoic } \\
\text { acid }\end{array}$ & 1.03397 & $6.50 \pm 0.87$ & $10.18 \pm 0.47$ & $12.21 \pm 1.97$ & $12.06 \pm 0.93$ \\
\hline & Ferulic acid & 1.01574 & $9.26 \pm 1.12$ & $12.35 \pm 0.52$ & $16.29 \pm 2.67$ & $16.22 \pm 1.98$ \\
\hline & Gluconic acid & 1.1915 & $11.93 \pm 1.17$ & $20.91 \pm 2.88$ & $17.50 \pm 3.94$ & $20.58 \pm 2.89$ \\
\hline & Glyceric acid & 1.41088 & $43.21 \pm 4.83$ & $54.70 \pm 2.83$ & $41.23 \pm 8.90$ & $44.25 \pm 4.11$ \\
\hline & Nicotinic acid & 1.0461 & $1.78 \pm 0.19$ & $2.27 \pm 0.38$ & $2.16 \pm 0.32$ & $3.24 \pm 0.33$ \\
\hline & Pyruvic acid & 0.832662 & $22.56 \pm 10.79$ & $12.03 \pm 3.13$ & $13.72 \pm 4.07$ & $10.20 \pm 1.51$ \\
\hline & Shikimic acid & 1.03754 & $40.69 \pm 3.01$ & $35.12 \pm 2.13$ & $25.98 \pm 4.36$ & $24.49 \pm 2.50$ \\
\hline & Sinapinic acid & 1.55069 & $18.06 \pm 1.38$ & $12.87 \pm 0.89$ & $19.58 \pm 3.27$ & $18.10 \pm 2.01$ \\
\hline & Suberyl glycine & 0.881479 & $17.81 \pm 6.66$ & $9.06 \pm 6.11$ & $2.56 \pm 1.11$ & $0.98 \pm 0.37$ \\
\hline & Succinic acid & 0.916328 & $105.28 \pm 5.58$ & $83.51 \pm 5.14$ & $79.00 \pm 7.30$ & $70.97 \pm 8.77$ \\
\hline & Tetronic acid & 1.01197 & $31.76 \pm 2.49$ & $28.47 \pm 0.99$ & $18.56 \pm 2.36$ & $9.37 \pm 1.54$ \\
\hline Others & Catechine & 0.853393 & $3.18 \pm 0.66$ & $2.19 \pm 0.54$ & $1.76 \pm 0.33$ & $1.77 \pm 0.54$ \\
\hline \multirow[t]{2}{*}{ Sugar } & Fructose & 1.34233 & $1280.18 \pm 309.92$ & $572.87 \pm 44.46$ & $583.52 \pm 61.67$ & $885.12 \pm 73.78$ \\
\hline & Glucose & 1.29197 & $133.76 \pm 48.83$ & $75.24 \pm 7.41$ & $75.90 \pm 13.83$ & $142.57 \pm 20.70$ \\
\hline \multirow[t]{3}{*}{ Polyol } & Galactosylglycerol & 1.53055 & $18.05 \pm 1.24$ & $8.99 \pm 0.73$ & $11.31 \pm 1.53$ & $16.29 \pm 1.84$ \\
\hline & Sorbitol & 1.22831 & $286.38 \pm 185.62$ & $93.69 \pm 7.52$ & $100.51 \pm 24.76$ & $258.18 \pm 16.60$ \\
\hline & Threitol & 1.22614 & $15.69 \pm 2.25$ & $6.34 \pm 0.53$ & $7.97 \pm 2.03$ & $4.26 \pm 0.27$ \\
\hline \multirow[t]{5}{*}{ Phosphoric acid } & Glucose-6-phosphate & 1.49567 & $5.09 \pm 0.84$ & $2.83 \pm 0.23$ & $4.03 \pm 0.55$ & $4.37 \pm 0.29$ \\
\hline & Fructose-6-phosphate & 1.1456 & $7.35 \pm 6.23$ & $1.44 \pm 0.18$ & $4.74 \pm 1.62$ & $6.46 \pm 1.11$ \\
\hline & Glyceric acid-3-phosphate & 1.66587 & $6.07 \pm 0.47$ & $2.64 \pm 0.42$ & $10.36 \pm 2.33$ & $8.09 \pm 1.82$ \\
\hline & Glycerol-3-phosphate & 1.02165 & $25.59 \pm 2.57$ & $19.82 \pm 1.95$ & $14.88 \pm 2.57$ & $15.39 \pm 1.76$ \\
\hline & Phosphoric acid & 1.10778 & $3804.99 \pm 215.58$ & $3288.56 \pm 122.31$ & $3384.19 \pm 444.48$ & $2075.88 \pm 130.26$ \\
\hline
\end{tabular}

Note: VIP is variable influence on projection values. Data are presented as mean \pm standard deviation.

\section{Differential metabolites during seed development}

According to the analysis of one-dimensional variance during metabolites of seed development (FDR $P<0.05)$ and VIP $>0.8,40$ metabolites were identified from a total 102 metabolites, including 14 amino acids, 13 organic acids, 2 sugars, 3 polyols and 5 phosphoric acids, which exhibited differential changes during seed development (Table 1). To show the regulated pattern of every key metabolic pathway, such as the glycolytic pathway, the metabolism of amino acids and the phenylpropanoid pathway, the metabolism was incorporated into a propositional metabolic pathway (Fig. 4).
As shown in Fig. 4, sucrose, as the precursor, was at a higher level at DAF 67 during seed-filling stage when compared with the other stages but the difference was not significant. However, glucose and fructose derived from the hydrolysis of sucrose were all more abundant. The glucose6-phosphate precursor presented in the two branches, which was glycolysis and the pentose phosphate pathway. On the one hand, glucose 6- phosphate in glycolysis entered into the pentose phosphate pathway and produced histidine. On the other hand, glutamic acid was produced by $\alpha$-ketoglutaric acid in the TCA cycle while entering into the pentose phosphate pathway and was further converted 
to lysine and proline. Glycerol-3-phosphate produced in glycolysis was converted to serine. One way in which the phenylpropanoid pathway generated tyrosine was through phosphoenolpyruvate in glycolysis, while the other portion of the pathway produced shikimic acid, phenylalanine, ferulic acid and sinapic acid. On one hand, pyruvic acid was the substrate needed to enter into amino acid metabolism pathway to produce valine, isoleucine, leucine and alanine. On the other hand, pyruvic acid acted as an intermediate product of amino acid metabolism by entering into TCA cycle to produce asparagine, threonine and glutamic acid.

\section{DISCUSSION}

Vegetable soybean, an environmentally-friendly food, has already been a secure, sanitary, nutritional and health-care food for modern consumers. As an export of vegetable soybean, in addition to yield traits, appearance quality is also a very important commodity (Zhang et al., 2007). As for appearance quality, despite the seeds that maintained a green color, the vegetable soybean pods appeared light yellow at DAF67, which did not meet the remarket demand of pods and seeds staying green (Han et al., 2003; Zhao et al., 2008). In order to obtain higher benefits to ensure higher yield and enhance selling potential, Mindou 6 soybeans were harvested between DAF61 and DAF67.

Such an organoleptic quality of vegetable soybean has been shown to be dependent on various factors, such as different varieties, the harvest stage, the duration between the harvest and processing data, and storage conditions (Czaikoski et al., 2013; Li et al., 2012a; Mozzoni et al., 2009; Song et al., 2013). Soluble sugar content in seeds is an important factor on eating quality (Zhang et al., 2015). Li et al (2012a) reported that sucrose was the highest content and accounted for $70 \%$ of soluble sugar at the edible stage of vegetable soybean. It was significantly and positively correlation between sucrose content and sweet taste scores (Wang and Wang, 2002). Meanwhile, the sucrose content was higher in fruit, fresh corn and vegetable soybean, so the effect of sucrose on the edible quality of fruits and vegetables has become the focus of recent research ( $\mathrm{Li}$ et al., 2012a; Li et al., 2012b; Cao et al., 2011). Among the nine sugars identified in Mindou 6 seeds, the tendency of glucose, fructose and sucrose was similar to that of total sugar, which decreased first and then increased gradually, especially at DAF61-DAF67, the period of rapid increase, when the highest sucrose content was reached. At the whole seed-filling stage, fructose content was about 6-10 times that of glucose. The sweetness of sucrose to the relative sweetness of carbohydrate is 1 , while fructose and glucose are 1.2-1.7 and 0.7-0.8, respectively (Bowers, 1992), namely compared to sucrose. Although the sucrose content was higher when compared with fructose, and fructose was higher when compared to sucrose in terms of sweetness, the results suggest that sweet property of vegetable soybean was highly related to the content of sucrose and fructose.

The amino acid content is an important factor, which mainly affects the eating quality aspect of umami of vegetable soybean (Silva et al., 2012; Zoldan et al., 2014). Masuda (1991) showed that the content of starch, sucrose, fructose, glutamic acid and alanine in vegetable soybean was higher, therefore, the flavor was enhanced. A lactic acid bacteria co-culture from paocai brine could significantly increase glutamic acid (umami), sucrose (sweetness), glycine (sweetness), lactic acid (sourness), and $\gamma$-aminobutyric acid in PB-paocai, which would endow it with important flavor features (Zhao et al., 2016). Of 21 kinds of amino acids identified from developing seeds, differential amino acids including alanine, glutamic acid, asparagine and 13 other types of amino acids played an important role in seed development and influenced eating quality. Studies have shown that asparagine, alanine and glutamic acid content of vegetable soybean have an important role in determining the quality of the vegetable soybean (Masuda, 1991; Zhao et al., 2016), which is consist with the results of this study. As the seeds matured, the amino acid content gradually decreased, and reached its lowest content at DAF67, which was 54.94\% lower than the amino acid levels displayed at DAF61.

As a product of plant primary metabolism and intermediates, organic acid not only regulated plant development, and responded to resistance to nutrient deprivation, metal stress, rhizosphere soil- plant microbe interaction, but organic acid was also one of the most important factors affecting fruit flavor and sensory quality (Wen et al., 2014; Liu et al., 2016; López-Bucio et al., 2001; Mattila and Hellstrom, 2007). Among 36 organic acids, citric acid, succinic acid, fumaric acid, malic acid and oxaloacetic acid were not only intermediate products belonging to the TCA cycle, but were also the substrate capable of producing the most amino acids. Relatively, the highest content of organic acid was citric acid, followed by malic acid and fumaric acid, which was consistent with the report of Song et al (2013). In addition, differential phenolic acids containing shikimic acid, ferulic acid and sinapic acid and other acids were identified by GC-MS, and played an important role in the flavor of vegetable soybean, which may be related to abundant secondary metabolites contained by soybean (Wu et al., 2008). Organic acid content in vegetable soybean seeds gradually increased and reached a climax at DAF67. Although our study analyzed the organic acid content of vegetable soybeans, the effect of organic acid content and composition on the flavor formation of vegetable soybean warrants further investigation. 
Polyol, also known as sugar alcohol, mainly acts as nutritional supplements in the food industry, and has certain physiological activity. Therefore, it is used to add sweetness and softness in sugar-free and diet food (Yang and Yang, 2003; Czaikoski et al., 2013). Polyol also affects eating quality of vegetable soybean, however, the content of polyols had no significant difference in the process of seed development, speculating it had little effect on the picking period.

Based on fresh pod weight, appearance quality and change in content of metabolites, the results showed that the fresh pod yield at the latter of the picking period had minimum change, however, the appearance quality worsened with as the picking period was further delayed. During DAF61-DAF67, the amino acid content decreased rapidly, on the contrary, sugar content dramatically increased and organic acid content gradually increased. Great consideration and achievement of marketing factors, such as yield, appearance quality, nutritional quality, eating quality, are necessary to ensure the highest economic benefit. As much, the harvesting of Mindou 6 beans should be completed during DAF61- DAF67, and the last date to harvest should be set to DAF67. Our results show that it is beneficial to sustain a longer podpicking period at R6 R7 to satisfy the market demand. Additional studies are necessary to determine the process by which vegetable soybean pod-picking can be extended, improvements can be exhibited regarding the expression of seed metabolites in the picking period, as well as the expression of metabolites in different cultivars.

\section{CONCLUSION}

During the seed-filling period of vegetable soybean Mindou 6 , amino acid, organic acid and sugar metabolites had a significant impact on its quality. Amino acids are known to contribute to the increase in nutrition and umami, while organic acids have been shown to improve its flavor, and sugar is known to increase its sweetness. With regard to yield, appearance quality, nutrition quality, eating quality and other factors, our studies found that the preferred pod-picking period was at DAF61-DAF67 and that the harvest must be completed before DAF67.

\section{ACKNOWLEDGMENTS}

This work was supported by National Key R\&D Program of China (2017YFD0101500), Fujian Provincial Major Scientific and Technological Special Project (2015NZ0002-3), Fujian Provincial Public Research Institutes for Basic Scientific Research Special Project (2016R1025-6), and Science and Technology Innovative Team in Fujian Academy of Agricultural Sciences
(STIT2017-2-6). We also thank the BioNovGene for the technical assistances.

\section{AUTHOR CONTRIBUTIONS}

L.G.Q.: Design and supervision of experiment and writing and reviewing the manuscript. Z.Y.M.: Collection of data and writing. H.R.F.: GC-MS work and statistical analysis of data. C.Y.H.: Field experiments.

\section{SUPPORTING INFORMATION}

File S1. The metabolite numbers were shown in File S1.

\section{REFERENCES}

Bowers, J. 1992. Food Theory and Application. Macmillan Pulishing Company, New York, NY.

Cao, Y. J., H. W. Zhao, X. H. Wang, W. W. Wei, L. Zhang, L. C. Wang and Y. J. Wang. 2011. Effects of potassium fertilization on yield, quality and sucrose metabolism of sweet maize. J. Plant Nutr. Fert. 17: 881-887.

Chebrolu, K. K., F. B. Fritschi, S. Ye, H. B. Krishnan, J. R. Smith and J. D. Gillman. 2016. Impact of heat stress during seed development on soybean seed metabolome. Metabolomics. 12: 1-14.

Czaikoski, K., R. S. Leite, J. M. G. Mandarino, M. C. Carrão-Panizzi, J. B. Silva and E. I. Ida. 2013. Canning of vegetable-type soybean in acidified brine: Effect of the addition of sucrose and pasteurisation time on color and other characteristics. Ind. Crop. Prod. 45: 472-476.

Duppong, L. M. and Hatterman-Valenti, H. 2013. Yield and quality of vegetable soybean cultivars for production in North Dakota. Horttechnology. 15: 896-900.

Fiehn, O. 2002. Metabolomics-The link between genotypes and phenotypes. Plant Mol. Biol. 48: 155-171.

Han, L. D., J. Y. Gai and J. X. Quu. 2003. A study on developmental process of pod and seed traits of summer-planted vegetable soybean and suitable pod picking period. Soybean Sci. 22: 202207.

Kopka, J., N. S. Schauer, C. Birkemeyer, B. Usadel, E. Bergmuller, P. Dormann, W. Weckwerth, Y. Gibon and M. Stitt. 2005. GMD@ CSB.DB: The golm metabolome database. Bioinformatics. 21: $1635-1638$.

Kortesniemi, M., A. L. Vuorinen, J. Sinkkonen, B. Yang, A. Rajala and H. Kallio. 2015. NMR metabolomics of ripened and developing oilseed rape (Brassica napus) and turnip rape (Brassica rapa). Food Chem. 172: 63-70.

Li, Y. S., D. Ming, Q. Y. Zhang, G. H. Wang, M. Hashemi and X. B. Liu. 2012a. Greater differences exist in seedprotein, oil, total soluble sugar and sucrose content of vegetable soybean genotypes (Glycine max (L.) Merrill) in Northeast China. Aust. J. Crop Sci. 6: 1681-1686.

Li, W. S, H. Yang, L. Shi, X. Y. Feng, C. S. Zhang and J. J. Yang. $2012 \mathrm{~b}$. Study on the correlation relationship between sweetness and sucrose, reducing sugars, soluble sugars in fruits. North. Hort. 1: 58-60.

Lisec, J, N. Schauer, J. Kopka, L. Willmitzer and A. R. Fernie. 2006. 
Gas chromatography mass spectrometry-based metabolite profiling in plants. Nat. Protoc. 1: 387-396.

Liu, R. L, Y. Y. Wang, G. Z. Qin and S. P. Tian. 2016. Molecular basis of 1-methylcyclopropene regulating organic acid metabolism in apple fruit during storage. Postharvest Biol. Tech. 117: 57-63.

López-Bucio, J, M. F. Nieto-Jacobo, V. Ramírez-Rodríguez and L. Herrera-Estrella. 2001. Organic acid metabolism in plants: From adaptive physiology to transgenic varieties for cultivation in extreme soils. Plant Sci. 160: 1-13.

Masuda, R. 1991. Quality requirement and improvement of vegetable soybean. In:, S .Proceedings of Vegetable soybean: Research needs for production and quality improvementAVRDC, Taibei, Taiwan, p. 92-102.

Mattila, P. and J. Hellstrom. 2007. Phenolic acids in potatoes, vegetables, and some of their products. J. Food Compos. Anal. 20: 152-160.

Mimura, M., C. J. Coyne, M. W. Bambuck and T. A. Lumpkin. 2007. SSR diversity of vegetable soybean [Glycine max (L.) Merr.]. Genet. Resour. Crop Evol. 54: 497-508.

Mozzoni, L. A, P. Chen, R. O. Morawicki, N. S. Hettiarachchy, K. R. Brye and A. Mauromoustakos. 2009. Quality attributes of vegetable soybean as a function of boiling time and condition. Int. J. Food Sci. Tech. 44: 2089-2099.

Sanchez, D. H., F. Schwabe, A. Erban, M. K. Udvardi and J. Kopka. 2012. Comparative metabolomics of drought acclimation in model and forage legumes. Plant Cell Environ. 35: 136-149.

Shepherd, L.V., P. Fraser and D. Stewart. 2011. Metabolomics: A second-generation platform for crop and food analysis. Bioanalysis. 3: 1143-1159.

Silva, J., S. Prudêncio, M. Carrão-Panizzi, C. Gregorut, F. Fonseca and L. Mattoso. 2012. Study of the flavour of soybean cultivars by sensory analysis and electronic tongue. Int. J. Food Sci. Tech. 47: 1630-1638.

Song, J. Y., G. H. An and C. J. Kim. 2003. Color, texture, nutrient contents, and sensory values of vegetable soybeans [Glycine $\max (\mathrm{L})$ Merrill] as affected by blanching. Food Chem. 83: 69-74.

Song, J., C. Liu, D. Li and Z. Gu. 2013. Evaluation of sugar, free amino acid, and organic acid compositions of different varieties of vegetable soybean (Glycine max [L.] Merr). Ind. Crop. Prod. 50: 743-749.

Tohge, T., T. Mettler, S. Arrivault, A. J. Carroll, M. Stitt and A. R. Fernie. 2011. From models to crop species: Caveats and solutions for translational metabolomics. Front. Plant Sci. 2: 61.
Vanholme, R., V. Storme, B. Vanholme, L. Sundin, J. H. Christensen, G. Goeminne, C. Halpin, A. Rohde, K. Morreel and W. Boerjan. 2012. A systems biology view of responses to lignin biosynthesis perturbations in Arabidopsis. Plant Cell. 24: 3506-3529.

Wang, D. Y. and Z. Q. Wang. 2002. Studies on the relationship between vegetable soybean eating quality and its components. J. Jinhua Polytech. 2: 15-17.

Wen, Y. Q., J. Cui, Y. Zhang, C. Q. Duan and Q. H. Pan. 2014. Comparison of organic acid levels and L-IdnDH expression in Chinese-type and European-type grapes. Euphytica. 196: 6376.

Wu, W., Q. Zhang, Y. Zhu, H. M. Lam, Z. Cai and D. Guo. 2008. Comparative metabolic profiling reveals secondary metabolites correlated with soybean salt tolerance. J. Agric. Food Chem. 56: 11132-11138.

Yang, Y. Z. and H. J. Yang. 2003. Application of functional sugar alcohol to the food industry. Global Food Ind. 7: 32-33.

Zhang, Q. Y., Y. S. Li, C. K. Liu, B. W. Tian, B. J. Xu and J. W. Mao. 2015. Key components of eating quality and their dynamic accumulation in vegetable soybean varieties [Glycine max (L.) Merr.]. Acta Agron. Sin. 41: 1692-1700.

Zhang, Q. Y., W. Y. Yang, Y. H. Li and X. W. Pan. 2007. Current status, production problem and prospects of vegetable soybean in China. Soybean Sci. 26: 950-954.

Zhang, Y. M., R. F. Hu and G. Q. Lin. 2013. Research advance on quality traits of vegetable soybean. Soybean Sci. 32: 698-702.

Zhang, Y. M., J. M. Zhao, M. J. Wang, H. Xing and J. Y. Gai. 2006. Genetic variance of nutritional quality of vegetbale soybean germplasm of Glycine max Merr. in southern China. Soybean Sci. 25: 239-243.

Zhao, J. M., C. Wang, Y. M. Zhang, H. H. Zhai, J. Y. Gai and H. Xing. 2008. Gentitc analysis and developmental speciality of sensory quality traits in vegetable soybean [Glycine $\max$ (L.) Merill]. Acta Hort. Sin. 35: 201-206.

Zhao, N., C. Zhang, Q. Yang, Z. Guo, B. Yang, W. Lu, D. Li, F. Tian, X. Liu, H. Zhang and W. Chen. 2016. Selection of taste markers related to lactic acid bacteria microflora metabolism for Chinese traditional paocai: A gas chromatography-mass spectrometry-based metabolomics approach. J. Agr. Food Chem. 64: 2415-2422.

Zoldan, S. M., G. D. S. Braga, F. J. Fonseca and M. C. CarrãoPanizzi. 2014. Electronic tongue system to evaluate flavor of soybean (Glycine max (L.) Merrill) genotypes. Braz. Arch. Biol. Tech. 57: 797-802. 
FILE S1. THE

METABOLITE NUMBERS

SHOWN IN FIG.2

\begin{tabular}{|c|c|c|c|c|c|}
\hline No. & Metabolite & No. & Metabolite & No. & Metabolite \\
\hline 1 & Tyrosine & 25 & 2,3-Dihydroxybutanedioic acid & 49 & Maltitol \\
\hline 2 & N-Acetyl glucosamine & 26 & Asparagine & 50 & 4-Hydroxy-3-methoxybenzoic acid \\
\hline 3 & Lysine & 27 & Shikimic acid & 51 & Ferulic acid \\
\hline 4 & Valine & 28 & Cysteine & 52 & Ribonic acid \\
\hline 5 & Isoleucine & 29 & Uracil & 53 & 3-Deoxy-arabino-hexaric acid \\
\hline 6 & Glutamine & 30 & Phosphoric acid & 54 & Glucopyranose \\
\hline 7 & Phenylalanine & 31 & Pyroglutamic acid & 55 & Monomethylphosphate \\
\hline 8 & beta-Alanine & 32 & Tetronic acid & 56 & Cellobiose \\
\hline 9 & Catechine & 33 & Adenine & 57 & Nicotinic acid \\
\hline 10 & Ethanolamine & 34 & Fumaric acid & 58 & Aconitic acid \\
\hline 11 & 2,5-Diaminovalerolactam & 35 & Malic acid & 59 & Citric acid \\
\hline 12 & Histidine & 36 & Malonic acid & 60 & Glucaric acid \\
\hline 13 & Proline & 37 & myo-Inositol & 61 & Galactinol \\
\hline 14 & Threonine & 38 & Threonic acid & 62 & Galactonic acid \\
\hline 15 & Leucine & 39 & Glycolic acid & 63 & Digalactosylglycerol \\
\hline 16 & Serine & 40 & myo-Inositol-1-phosphate & 64 & Sucrose \\
\hline 17 & Alanine & 41 & Benzoic acid & 65 & Isomaltose \\
\hline 18 & Succinic acid & 42 & Uridine & 66 & Glucose \\
\hline 19 & Suberyl glycine & 43 & Lactic acid & 67 & Sorbitol \\
\hline 20 & Homoserine & 44 & 4-hydroxy-Butyric acid & 68 & Galactosylglycerol \\
\hline 21 & Threonic acid-1,4-lactone & 45 & 2-Aminoadipic acid & 69 & Fructose-6-phosphate \\
\hline 22 & 4-Aminobutyric acid & 46 & 2-Hydroxyglutaric acid & 70 & Fructose \\
\hline 23 & Threitol & 47 & Glyceric acid & 71 & Spermidine \\
\hline 24 & Glycerol-3-phosphate & 48 & Gluconic acid & 72 & Glucose-6-phosphate \\
\hline
\end{tabular}

